\title{
Use of Large Retrospective Databases to Guide Tumor Staging Criteria
}

\author{
D. A. Dominguez, MD, and L. W. Thornblade, MD \\ Department of Surgery, University of California, Oakland, CA
}

The presence of hypoxia within solid tumors is a welldocumented phenomenon that plays a complex role in tumorigenesis both through direct effects promoting coagulative necrosis, and more indirect effects on gene expression mediated through hypoxia-inducible factor- $1 \alpha$ $($ HIF-1 $\alpha) .{ }^{1}$ Hypoxia and resultant necrosis often are observed in aggressive tumors, giving rise to the theory that rapid proliferation causes tumors to outgrow their own blood supply. Intratumoral hypoxia is reflected on histopathologic analysis by the presence of tumor necrosis, which has been associated with aggressive phenotypes and poor long-term outcomes in a variety of tumor types including breast, pancreatic, renal cell, and bladder cancer. $^{2-5}$

In this issue of Annals of Surgical Oncology, Tsilimigras et al. examine the prognostic implications of the presence of tumor necrosis on histopathologic examination from 757 patients with intrahepatic cholangiocarcinoma using data maintained by the International Intrahepatic Cholangiocarcinoma Study Group, which collects data from 15 major hepatobiliary centers around the world. ${ }^{6}$ Not only did they find the presence of necrosis to be associated with other risk factors for higher risk disease, such as tumor size, higher preoperative CA19-9, and poor/undifferentiated grade, but also that it was associated with both a lower median 5-year recurrence-free survival (RFS) and overall survival (OS) in patients with tumors staged as $\mathrm{T} 1$ by current 8th edition American Joint Committee on Cancer

(C) Society of Surgical Oncology 2022

First Received: 3 February 2022

Accepted: 5 February 2022;

Published Online: 2 March 2022

D. A. Dominguez, MD

e-mail: ddominguez@alamedahealthsystem.org
(AJCC) criteria. The authors propose new staging criteria for $\mathrm{T} 1$ tumors, in which the presence of necrosis would upstage a T1a to a $\mathrm{T} 1 \mathrm{~b}$, and subsequently a $\mathrm{T} 1 \mathrm{~b}$ to a $\mathrm{T} 2$ tumor. Using multivariable survival regression, their staging system demonstrated an improved model fit in predicting patient survival outcomes compared with the existing staging system.

The development of international multicenter databases has been instrumental to the development and validation of staging systems, especially in relatively rare tumors, such as intrahepatic cholangiocarcinoma. However, there are limitations to databases that are not prospectively maintained, including incongruent and missing data. Indeed, of the patients examined by the authors, $32 \%$ of the initial cohort $(n=1,143)$ were missing either follow-up data $(n=$ 193) or pathologic data $(n=174)$ —a significant proportion of the studied population. Ideally, staging systems developed from examination of one database would be validated by application in another database, although existence of parallel databases containing similar variables can make this often impossible. This is one potential explanation for why to date, tumor necrosis has not yet been incorporated into any AJCC TNM staging criteria for other pathologies. An example demonstrating a potential pitfall of large databases was seen after the development of the AJCC 8th edition for gallbladder carcinoma in which $\mathrm{T} 2$ tumors were subdivided into $\mathrm{T} 2 \mathrm{a}$ and $\mathrm{T} 2 \mathrm{~b}$ based on tumor location: peritoneal side versus hepatic side. This was in part based on data from an international multicenter study of 437 patients, which showed a worse prognosis in patients with hepatic sided T2 tumors compared with peritoneal sided T2 tumors. ${ }^{7}$ Interestingly, when examined in a cohort of 1,251 patients with $\mathrm{T} 2$ gallbladder carcinoma using the National Cancer Database (NCDB), a retrospective database of patients in the United States, tumor location was not associated with differential survival on multivariable cox 
regression analysis. ${ }^{8}$ This illustrates the importance of cross-validation of staging systems, ideally with more than one dataset, in particular when data are collected retrospectively, when feasible.

Two additional points of consideration before incorporation of a histopathologic factor into a staging system are 1) the possible effect of treatment on that factor, and 2) the collection and availability of the factor for application. In the case of tumor necrosis, treatment strategies, such as portal vein embolization, radiation, and neoadjuvant chemo- and immunotherapy, have the potential for altering the inherent content of necrosis in a tumor, thereby influencing the proposed stage. As to the availability of the factor for analysis, any staging criteria must be universally collected to allow for wide applicability. Because tumor necrosis is currently not mentioned in the most recent AJCC staging manual, it is likely not routinely collected as a measure uniformly at institutions worldwide, another potential explanation why it is not yet part of any AJCC staging. ${ }^{9}$

Regardless of their inherent limitations, international multicenter databases remain a powerful tool for our understanding of rare cancers. Implementation should be done with consideration of potential confounding factors and critical validation.

\section{DECLARATIONS}

DISCLOSURE The authors declare no conflicts of interest.

\section{REFERENCES}

1. Vaupel P, Mayer A. Hypoxia in cancer: significance and impact on clinical outcome. Cancer Metastasis Rev. 2007;26(2):225-39. h ttps://doi.org/10.1007/S10555-007-9055-1.
2. Gilchrist KW, Gray R, Fowble B, Tormey DC, Taylor SG IV. Tumor necrosis is a prognostic predictor for early recurrence and death in lymph node-positive breast cancer: a 10-year follow-up study of 728 Eastern Cooperative Oncology Group patients. J Clin Oncol. 1993;11(10):1929-35. https://doi.org/10.1200/JCO.1993.1 1.10.1929.

3. Hiraoka $\mathrm{N}$, Ino $\mathrm{Y}$, Sekine $\mathrm{S}$, et al. Tumour necrosis is a postoperative prognostic marker for pancreatic cancer patients with a high interobserver reproducibility in histological evaluation. Br J Cancer. 2010;103(7):1057-65. https://doi.org/10.1038/SJ.BJ C.6605854.

4. Syed M, Loya A, Hameed M, Akhtar N, Mushtaq S, Hassan U. Prognostic significance of percentage necrosis in clear cell renal cell carcinoma. Am J Clin Pathol. Published online October 13, 2021.https://doi.org/10.1093/AJCP/AQAB136

5. Ord JJ, Agrawal S, Thamboo TP, et al. An investigation into the prognostic significance of necrosis and hypoxia in high grade and invasive bladder cancer. J Urol. 2007;178(2):677-82. https://doi. org/10.1016/J.JURO.2007.03.112.

6. Tsilimigras DI, Ejaz A, Cloyd J, et al. Tumor necrosis impacts prognosis of patients undergoing resection for $\mathrm{T} 1$ intrahepatic cholangiocarcinoma. Ann Surg Oncol. 2022. https://doi.org/10.12 45/s10434-022-11462-y.

7. Shindoh J, de Aretxabala X, Aloia TA, et al. Tumor location is a strong predictor of tumor progression and survival in T2 gallbladder cancer: an international multicenter study. Ann Surg. 2015;261(4):733. https://doi.org/10.1097/SLA.0000000000000728.

8. Lafaro K, Blakely AM, Melstrom LG, et al. Prognostic impact of tumor location in resected gallbladder cancer: a national cohort analysis. J Surg Oncol. 2020;122(6):1084-93. https://doi.org/10. 1002/JSO.26107.

9. Amin MB, Edge SB, Greene FL, et al. American Joint Committee on Cancer (AJCC). AJCC Cancer Staging Manual. AJCC Cancer Staging Manual. Published online 2017:211-212.

Publisher's Note Springer Nature remains neutral with regard to jurisdictional claims in published maps and institutional affiliations. 